\title{
Further Investigation of the Negative Association between Hypertension and Peripheral Neuropathy in the Elderly: An Oklahoma Physicians Resource/ Research Network (OKPRN) Study
}

\author{
Dong Yung Cho, MD, James W. Mold, MD, and Michelle Roberts, BS
}

Background: In a previous cross-sectional study of age-associated peripheral neuropathy (AAPN), we found that a history of hypertension was protective. The purpose of this study, conducted in the same cohort, was to investigate further this association in the same subjects and in a comparison group of older subjects with diabetes mellitus.

Methods: Baseline data from $\mathbf{5 8 4}$ subjects involved in a longitudinal study of primary care patients 65 years of age and older, with no history of 10 medical conditions known to cause peripheral neuropathy, were analyzed for associations between peripheral neuropathy by examination and history of hypertension, number of antihypertensive medications, systolic blood pressure, diastolic blood pressure, pulse pressure, and orthostatic hypotension. In addition, we examined associations between neuropathy at baseline and use of specific classes of antihypertensive medications and non-steroidal anti-inflammatory agents (NSAIDs) in year 3. The analyses were repeated in 110 subjects with diabetes mellitus.

Results: History of hypertension, but not the other hypertension-related variables, was negatively associated (OR, 0.60; 95\% CI: 0.40 to 0.90$)$ with AAPN after controlling for age and body mass index (BMI). In 287 subjects evaluated in the 3rd year of the study, $\beta$-blocking agents (OR, 3.56; 95\% CI: 1.58 to 8.03) and NSAIDs (OR, 2.65; $95 \%$ CI: 1.37 to 5.10) were positively associated with AAPN. In subjects with diabetes mellitus, a history of hypertension was again protective, but current pulse pressure (OR, 1.03; 95\% CI: 1.00 to 1.05) was a positive predictor of peripheral neuropathy. There were interesting interactions between pulse pressure and military service and pulse pressure and BMI in diabetic patients.

Conclusions: The negative association between hypertension and AAPN remains unexplained. The positive association between pulse pressure and neuropathy in diabetic subjects supports findings from previous studies and suggests that AAPN and the neuropathy of diabetes may be distinct entities. The incidental finding of an association between NSAIDs and AAPN is concerning and should be investigated further. (J Am Board Fam Med 2006;19:240-50.)

The prevalence of peripheral neuropathy increases rapidly beyond age 65 . In many if not most cases, the cause is unknown. In a group of primary care patients over 65 years of age, we found that peripheral neuropathy could be detected by physical ex-

Submitted 8 April 2005; revised 29 June 2005; accepted 5 July 2005.

From the Department of Family and Preventive Medicine, University of Oklahoma Health Sciences Center, Oklahoma City, OK.

Funding: The OKLAHOMA Studies cohort studies were made possible by a grant from the Presbyterian Health Foundation of Oklahoma. The work of DC was supported by the Konkuk University (Seoul, Korea).

Conflict of interest: none declared.

Corresponding author: James W. Mold, MD, MPH, Department of Family and Preventive Medicine, University of Oklahoma Health Sciences Center, $900 \mathrm{NE} 10^{\text {th }}$ Street, Oklahoma City, OK 73104 (E-mail: james-mold@ouhsc.edu). amination in $31 \%$, two thirds of whom had no history of 10 medical conditions known to cause it. ${ }^{1}$ Because age-associated peripheral neuropathy (AAPN) is so common, it has generally been considered to be of no clinical importance, and so it has not been well studied. However, it may not be completely benign. In the aforementioned study, subjects with peripheral neuropathy were more likely to report a variety of symptoms (numbness, pain, restless legs) and functional difficulties (trouble walking, trouble with balance), and they had lower quality of life scores even after controlling for underlying medical conditions. ${ }^{1}$

The cause or causes of AAPN remain a mystery. One theory is that it involves reductions in the bioavailability of neurotropic growth factors. ${ }^{2,3}$ 
Others believe it can be attributed to unrecognized diabetes (personal communication with local experts) or that it is a manifestation of microvascular disease (ie, ischemic neuropathy).

Variables associated with peripheral neuropathy in our previous study included increasing age, increasing body mass index (BMI), prior military service, and a history of diabetes, rheumatoid arthritis, or vitamin $\mathrm{B}_{12}$ deficiency. A history of hypertension was protective. ${ }^{1}$ This was inconsistent with studies of subjects with diabetic neuropathy, which have suggested that hypertension may be an aggravating factor. ${ }^{4,5}$ Two studies of older subjects with idiopathic peripheral neuropathy also found a positive association between hypertension and neuropathy. ${ }^{6,7}$ However, one of them was done in a population of subjects referred to a neurologist, whereas the other examined only symptomatic subjects and did not control for BMI.

The purpose of this study was to further investigate the negative association between hypertension, hypertension-related variables, and peripheral neuropathy in older subjects with no obvious medical cause for their neuropathy, and in a group of older subjects with diabetes to gain additional insight into the cause or causes of AAPN. We were specifically interested to know whether a history of hypertension or current blood pressure elevation was the stronger predictor of AAPN and whether any medications commonly used in patients with hypertension might be neuroprotective. In addition, we were looking for further evidence of similarities and differences between AAPN and diabetic peripheral neuropathy, because one theory has been that AAPN is simply a milder form of diabetic neuropathy in those not yet diagnosed with full-blown diabetes.

\section{Methods}

\section{Data Collection}

All analyses involved data from the Oklahoma Longitudinal Assessment of Health Outcomes in Mature Adults (OKLAHOMA) Studies cohort. The methods used to recruit and enroll subjects into the cohort have been described previously. ${ }^{1}$ Subjects over 65 years of age were recruited from the practices of 23 family physician members of the Oklahoma Physicians Resource/Research Network (OKPRN), a primary care practice-based research network. Physicians generated lists of patients $>65$ years old seen by them within the prior 18 months $(n=4762)$. From these lists, they indicated those who were no longer their patients, currently living in nursing homes $(\mathrm{n}=213)$, too confused to sign informed consent $(\mathrm{n}=165)$, or deceased $(\mathrm{n}=337)$. An additional 815 had switched physicians, leaving 3232 potentially eligible patients. The practices sent a letter to these patients explaining the study and inviting them to participate. This was followed in 2 weeks by a telephone call from the project coordinator. The coordinator was able to reach 1977 patients, of which 11 were disqualified because of cognitive impairment (unable to understand the purpose of the call or the study).

Eligible patients who agreed to participate $(\mathrm{n}=$ 853) were asked to complete a questionnaire, which included demographic information, health habits, medical conditions, symptoms, functional status, and several health-related quality-of-life instruments. Those who declined to participate were asked to provide information about why they were not interested, their current age and race, and their self-perceived state of health. Participants were more likely than non-participants to be male (43\% vs. $37 \% ; P=.002)$, younger $(P<.0001)$, better educated $(P=<.0001)$, and in better health $(P=$ $<.0001)$. Blacks tended to be less willing to participate $(P=.06){ }^{1}$

At the enrollment visit, 2 research nurses reviewed the completed questionnaires for missing data and conducted focused physical examinations including weight, height, blood pressure, and pulse lying and standing, and examinations of fine touch in the feet, position of the great toe, vibration at the medial malleoli, and deep tendon reflexes at the ankles.

Fine touch was measured using a 5.07 SemmesWeinstein monofilament. With eyes closed, the subject was asked to identify when the filament touched the sole of the foot at each of eight different sites (1st, $3 \mathrm{rd}$, and 5 th toes; $1 \mathrm{st}, 3 \mathrm{rd}$, and 5 th metatarsals; and medial and lateral sole at the midfoot). To be considered intact, the subject had to be correct at 6 or more of the 8 sites. Position sensation was tested by asking the subject, with eyes still closed, to indicate the direction (up or down) the examiner manually moved their great toe while holding it lateromedially between thumb and first finger. To be considered intact, the subject had to be correct on 4 of 5 movements. 
Vibratory sense was tested using a $\mathrm{C}_{128}$ tuning fork, struck with moderate force against the examiner's thenar eminence and applied to the subject's medial malleoli. Intact vibratory sensation was defined as perception of vibration, buzzing, or tingling. Ankle reflexes were tested using a standard triangular rubber-headed reflex hammer. Subjects were seated with knees bent at $90^{\circ}$. With the ankle also at $90^{\circ}$, they were asked to gently press the sole of the foot into the examiner's hand as the Achilles tendon was tapped with moderate force. Any detectable reflexive response was considered to be an intact reflex. Peripheral neuropathy was defined as bilateral absence of one or more of the four sensory functions.

The nurses were trained by a neurologist to perform the peripheral neurological examination. Once they were comfortable, for quality control purposes, they separately examined the same group of 25 study enrollees, blinded to the other's conclusions. Their findings were identical for all neurological findings except ankle reflex where there was disagreement on 3 of the 25 . They were subsequently retrained on detection of ankle reflexes.

The same groups of subjects were re-enrolled annually between January of 2000 and December of 2004, with somewhat different sets of data collected each time. In year 3, subjects were required to bring to their evaluations all their medications taken more often than once every week. This was the only time during the 5-year study that information on medications was obtained. These were recorded and categorized by pharmaceutical class according to the system used in the Physicians Desk Reference.

\section{Analyses}

Our analytic strategies were designed to answer the following questions: 1) After controlling for other associated variables, is a history of hypertension negatively associated with AAPN? 2) If so, does the association exist only for historical hypertension or for current blood pressure as well, and if both, which is the stronger association? 3) If a negative association exists, could use of a particular type of antihypertensive agent or agents explain the association? 4) Are the patterns of association between hypertension and neuropathy similar for the "idiopathic" group and for patients with diabetes?

We examined associations between several hypertension-related variables and peripheral neurop- athy in two groups of subjects: those with no selfreported history of ten medical conditions known to cause neuropathy (diabetes, rheumatoid arthritis, lupus, scleroderma, $\mathrm{B}_{12}$ deficiency, chronic hepatitis, chronic renal failure, Crohn's disease, sarcoidosis, or hereditary neuropathy), and those with a self-reported history of diabetes mellitus. Hypertension-related variables included a history of hypertension, baseline systolic, diastolic, and pulse pressure lying for $5 \mathrm{~min}$ and standing for $1 \mathrm{~min}$ and $5 \mathrm{~min}$, baseline orthostatic drop in systolic pressure, and use of any of the following classes of antihypertensive medications in year 3: thiazide diuretics, $\beta$-adrenergic blockers ( $\beta$-blockers), $\alpha$-adrenergic blockers, calcium channel blockers, angiotensin-converting enzyme inhibitors, and vasodilators. We created a new variable, number of antihypertensive medications, which we then dichotomized (0 to 1 antihypertensive medications versus 2 or more). We also examined the use of nonsteroidal anti-inflammatory agents (NSAIDs) as a potential confounding variable. Variables found to be associated with peripheral neuropathy in our previous study (age, BMI, military service) were also included. In addition, we examined relationships between hypertension-related variables and the individual neurological deficits. We looked for potential interactions between variables remaining in the final regression models.

We examined bivariate associations between categorical variables using the $\chi^{2}$ test. Differences between means of continuous variables were examined using Student's independent $t$ test. Multivariate logistic regression models were created to simultaneously examine associations between peripheral neuropathy, the dependent variable, and variables found to be potentially associated with peripheral neuropathy in bivariate analyses $(P<$ .2). A backward elimination technique was used to identify the most parsimonious models, based on stability of deviance. Because of the high degree of correlation between the hypertension-related independent variables, we examined individual associations between each variable and peripheral neuropathy as well as their contributions when considered as a group. Only those variables found to be most strongly and consistently associated with peripheral neuropathy were considered in the regression models. All statistical analyses were performed using Statistix 8 (Analytical Software, Tallahassee FL). 


\begin{tabular}{|c|c|c|c|c|c|c|}
\hline Continuous Variables & $\begin{array}{c}\mathrm{PN}(-) \mathrm{N}=436 \\
\text { Mean }(\mathrm{SD})\end{array}$ & \multicolumn{3}{|c|}{$\begin{array}{c}\mathrm{PN}(+) \mathrm{N}=148 \\
\text { Mean }(\mathrm{SD})\end{array}$} & $\mathrm{T}$ & $P$ Value \\
\hline Age & $72.68(5.50)$ & \multicolumn{3}{|c|}{$75.70(6.55)$} & -5.02 & $<.0001$ \\
\hline BMI & $28.12(5.01)$ & \multicolumn{3}{|c|}{$29.07(5.86)$} & -1.76 & .08 \\
\hline Orthostatic BP & $13.46(47.76)$ & \multicolumn{3}{|c|}{$13.11(11.33)$} & 0.14 & .89 \\
\hline Pulse pressure & $65.24(17.89)$ & \multicolumn{3}{|c|}{$65.35(17.96)$} & -0.07 & .95 \\
\hline Diast $\mathrm{BP}(5$ Rest $)$ & $76.17(10.90)$ & \multicolumn{3}{|c|}{$75.01(12.12)$} & 1.02 & .31 \\
\hline Diast BP(1 Stand $)$ & $75.85(11.16)$ & \multicolumn{3}{|c|}{$73.94(13.52)$} & 1.54 & .12 \\
\hline Diast BP(5 Stand) & $76.30(12.53)$ & \multicolumn{3}{|c|}{$74.65(13.30)$} & 1.31 & .19 \\
\hline Syst BP(5 Rest) & $141.55(20.47)$ & \multicolumn{3}{|c|}{$140.36(23.20)$} & 0.55 & .58 \\
\hline Syst BP(1 Stand $)$ & $138.73(51.72)$ & \multicolumn{3}{|c|}{$135.00(23.99)$} & 1.17 & .24 \\
\hline Syst BP(5 Stand $)$ & $136.51(22.67)$ & \multicolumn{3}{|c|}{$136.60(23.34)$} & -0.07 & .95 \\
\hline Number of visits & $4.32(4.05)$ & \multicolumn{3}{|c|}{$4.68(3.60)$} & -1.01 & .31 \\
\hline Categorical Variables & State & $\mathrm{PN}(-)$ & \multicolumn{2}{|l|}{$\mathrm{PN}(+)$} & $x^{2}$ & $P$ Value \\
\hline \multirow[t]{2}{*}{ Gender } & Female & 247 & \multicolumn{2}{|l|}{77} & 0.96 & .32 \\
\hline & Male & 189 & \multicolumn{2}{|l|}{71} & & \\
\hline \multirow[t]{2}{*}{ Hypertension } & $(-)$ & 240 & \multicolumn{2}{|l|}{94} & 3.24 & .07 \\
\hline & $(+)$ & 196 & \multicolumn{2}{|l|}{54} & & \\
\hline \multirow[t]{2}{*}{ Military service } & $(-)$ & 293 & \multicolumn{2}{|l|}{90} & 2.00 & .16 \\
\hline & $(+)$ & 143 & \multicolumn{2}{|l|}{58} & & \\
\hline \multicolumn{7}{|l|}{ Logistic Regression } \\
\hline Predictor Variables & Coefficient & \multicolumn{2}{|l|}{ Standard Error } & $P$ Value & & Deviance $(P$ Value \\
\hline Age & 0.10 & 0.02 & \multicolumn{3}{|c|}{$<.0001$} & \\
\hline BMI & 0.06 & 0.02 & & .001 & & 613.48 \\
\hline Hypertension & -0.51 & 0.21 & & .01 & & $(P=.11)$ \\
\hline
\end{tabular}

* PN, peripheral neuropathy; BMI, body mass index $\left(\mathrm{kg} / \mathrm{m}^{2}\right)$; pulse pressure, systolic blood pressure - diastolic blood pressure; Diast $\mathrm{BP}(1 \mathrm{St})$, diastolic blood pressure $1 \mathrm{~min}$ after standing position; Diast $\mathrm{BP}(5 \mathrm{Res})$, diastolic blood pressure 5 min after rest; Diast $\mathrm{BP}(5 \mathrm{St})$, diastolic blood pressure 5 min after standing position; Syst $\mathrm{BP}(1 \mathrm{St})$, systolic blood pressure 1 min after standing position; Syst BP(5Res), systolic blood pressure 5 min after rest; Syst BP(5St), systolic blood pressure 5 min after standing position; Orthostatic $\mathrm{BP}$, Syst BP(1St) - Syst BP(5Res) ; number of visits, number of clinic visits; (+), positive history; (-), negative history.

The two-tailed significance level for the models was set at 0.05 .

\section{Results \\ General}

The original OKLAHOMA Studies cohort consisted of 854 subjects. Nine were excluded because we could not determine their peripheral neuropathy status (eg, amputation). A total of 261 reported having one or more of the medical conditions associated with neuropathy leaving 584 in the primary study population. The comparison group included 110 subjects who gave a history of diabetes mellitus.

Subjects in the primary study population who had peripheral neuropathy were significantly older $(P<.0001)$ but did not differ with regard to mean
BMI, systolic pressure, diastolic blood pressure, pulse pressure, or orthostatic fall in systolic pressure from those without peripheral neuropathy though diastolic blood pressures tended to be lower in subjects with peripheral neuropathy (Table 1).

Only 287 of 584 subjects remained in the study in year 3 when medications were documented. The negative association between hypertension and neuropathy was no longer seen in those remaining, most likely because subjects with peripheral neuropathy but not hypertension dropped out of the study at a higher rate than subjects with peripheral neuropathy and hypertension $(P=.008)$. There was no difference in the rate at which subjects without peripheral neuropathy dropped out based on presence or absence of hypertension. The reasons given for dropping out before the third year 
Table 2. Characteristics of Subjects Who Had No Diseases Known to Cause Peripheral Neuropathy and Who Were Still Enrolled in the Third Year of the Study $(\mathrm{N}=287)$

\begin{tabular}{|c|c|c|c|c|c|}
\hline Continuous Variables & $\begin{array}{c}\mathrm{PN}(-) \mathrm{N}=225 \\
\text { Mean }(\mathrm{SD})\end{array}$ & \multicolumn{2}{|c|}{$\begin{array}{c}\mathrm{PN}(+) \mathrm{N}=62 \\
\text { Mean (SD) }\end{array}$} & $\mathrm{T}$ & $P$ Value \\
\hline Age & $72.90(5.56)$ & \multicolumn{2}{|c|}{$72.77(5.16)$} & 0.17 & .87 \\
\hline BMI & $28.11(4.79)$ & \multicolumn{2}{|c|}{$29.93(6.10)$} & -2.16 & .03 \\
\hline Pulse pressure & $64.13(17.93)$ & \multicolumn{2}{|c|}{$63.81(15.15)$} & 0.14 & .89 \\
\hline Otho BP & $11.50(0.92)$ & \multicolumn{2}{|c|}{$13.60(11.06)$} & -1.25 & .21 \\
\hline Diast BP(5 Rest) & $75.03(10.43)$ & \multicolumn{2}{|c|}{$75.45(11.97)$} & -0.25 & .80 \\
\hline Diast BP(1 Stand) & $74.55(11.07)$ & \multicolumn{2}{|c|}{$75.90(14.21)$} & -0.69 & .49 \\
\hline Diast BP(5 Stand) & $74.69(12.38)$ & \multicolumn{2}{|c|}{$76.30(13.29)$} & -0.85 & .40 \\
\hline Syst BP(5 Rest) & $139.16(19.22)$ & \multicolumn{2}{|c|}{$139.26(21.32)$} & -0.03 & .97 \\
\hline Syst BP(1 Stand) & 133.25 (21.94) & \multicolumn{2}{|c|}{$135.05(23.00)$} & -0.55 & .59 \\
\hline Syst BP(5 Stand) & $134.44(20.46)$ & \multicolumn{2}{|c|}{$135.41(21.41)$} & -0.32 & .75 \\
\hline Categorical Variables & State & $\mathrm{PN}(-)$ & $\mathrm{PN}(+)$ & $\chi^{2}$ & $P$ Value \\
\hline \multirow[t]{2}{*}{ Gender } & Female & 124 & 30 & 0.88 & .35 \\
\hline & Male & 101 & 32 & & \\
\hline \multirow[t]{2}{*}{ Hypertension } & $(-)$ & 134 & 37 & 0.00 & .99 \\
\hline & $(+)$ & 91 & 25 & & \\
\hline \multirow[t]{2}{*}{ ACE-I \& ARB* } & $(-)$ & 181 & 49 & 0.06 & .81 \\
\hline & $(+)$ & 44 & 13 & & \\
\hline \multirow[t]{2}{*}{$\alpha$ blocking agents } & $(-)$ & 207 & 56 & 0.18 & .67 \\
\hline & $(+)$ & 18 & 6 & & \\
\hline \multirow[t]{2}{*}{$\beta$ blocking agents } & $(-)$ & 209 & 49 & 10.27 & .001 \\
\hline & $(+)$ & 16 & 13 & & \\
\hline \multirow[t]{2}{*}{ Calcium channel block } & $(-)$ & 203 & 55 & 0.12 & .73 \\
\hline & $(+)$ & 22 & 7 & & \\
\hline \multirow[t]{2}{*}{ Diuretics } & $(-)$ & 187 & 48 & 1.06 & .30 \\
\hline & $(+)$ & 38 & 14 & & \\
\hline \multirow[t]{2}{*}{ Vasodilators } & $(-)$ & 218 & 62 & 1.98 & .16 \\
\hline & $(+)$ & 7 & 0 & & \\
\hline \multirow[t]{2}{*}{ NSAIDs } & $(-)$ & 190 & 42 & 8.75 & .003 \\
\hline & $(+)$ & 35 & 20 & & \\
\hline \multirow[t]{2}{*}{ Military service } & $(-)$ & 146 & 36 & 0.98 & .32 \\
\hline & $(+)$ & 79 & 26 & & \\
\hline \multirow[t]{3}{*}{ Number of medications } & 0 & 138 & 30 & 3.61 & .16 \\
\hline & 1 & 45 & 15 & & \\
\hline & 2 or more & 42 & 17 & & \\
\hline \multicolumn{6}{|l|}{ Logistic Regression } \\
\hline Predictor Variables & Coefficient & Standard Error & $P$ Value & & Deviance $(P$ Value $)$ \\
\hline BMI & 0.05 & 0.03 & .07 & & \\
\hline$\beta$-blocking agents & 1.29 & 0.42 & .002 & & 277.20 \\
\hline NSAID & 0.84 & 0.34 & .01 & & $(P=.52)$ \\
\hline
\end{tabular}

* ACE-I \& ARB, angiotensin-converting enzyme inhibitor and angiotensin receptor blocker; BMI, body mass index; NSAIDs, nonsteroidal antiinflammatory drugs.

were no different for subjects with peripheral neuropathy and hypertension than for those with peripheral neuropathy and no hypertension, and subjects who dropped out reported the same number of visits per year as those who remained in the study.
Subjects with No History of Diseases Known to Cause Peripheral Neuropathy

In this group, bivariate associations between peripheral neuropathy and age, history of military service, history of hypertension, standing diastolic 
Table 3. Associations between Use of $\beta$ Blockers or NSAIDs* and Specific Neurological Deficits after Controlling for Age and BMI in Subjects Who Had no Diseases Known to Cause Peripheral Neuropathy and Who Were Still Enrolled in Year 3 of the Study $(N=287) *$

\begin{tabular}{ccccc}
\hline & Predictor Variables & Coefficient & Standard Error & $P$ Value \\
\hline PN(ankle) $\dagger$ & BETA & 1.24 & 0.43 & 0.004 \\
$(\mathrm{~N}=50)$ & NSAID & 0.50 & 0.37 & 0.18 \\
$\mathrm{PN}($ touch) & BETA & 0.23 & 0.80 & 0.77 \\
$(\mathrm{~N}=16)$ & NSAID & 1.21 & 0.54 & 0.02 \\
$\mathrm{PN}($ position $)$ & BETA & 1.90 & 0.97 & 0.05 \\
$(\mathrm{~N}=5)$ & NSAID & 1.93 & 0.96 & 0.04 \\
$\mathrm{PN}($ vibration $)$ & BETA & 1.44 & 0.95 & 0.13 \\
$(\mathrm{~N}=7)$ & NSAID & 3.27 & 1.07 & 0.002 \\
\hline
\end{tabular}

* Statistical analysis was done by logistic regression.

† PN(ankle), subjects who had deficit in ankle reflex; PN(touch), subjects who had deficit in touch sensation; PN(position), subjects who had deficit in position sensation; PN(vibration), subjects who had deficit in vibration sensation.

blood pressure after $1 \mathrm{~min}$, standing diastolic blood pressure after $5 \mathrm{~min}$ had values of $P<.2$. Not surprisingly, history of hypertension was associated with systolic, diastolic, and pulse pressure. History of hypertension was the most representative and was the only variable considered in the logistic regression model. The final model (Table 1) included age (OR, 1.10/year; 95\% CI, 1.07 to 1.14 ), BMI (OR, 1.06/unit; 95\% CI, 1.03 to 1.11 ), and history of hypertension (OR, $0.60 ; 95 \%$ CI, 0.40 to $0.90)$. There were no significant interactions (Table 1).

For the 287 subjects remaining in the third year of the study, we analyzed associations between antihypertensive medications and NSAIDs, and peripheral neuropathy. Table 2 contains the bivariate associations and the regression model. After controlling for other variables, $\beta$ blockers (OR, 3.56; 95\% CI, 1.58 to 8.03 ) and NSAIDs (OR, 2.65; $95 \%$ CI, 1.37 to 5.10 ) were associated with peripheral neuropathy. There were no significant interactions (Table 2). $\beta$ blockers were associated with loss of ankle reflexes only, whereas NSAIDs were associated with the other 3 deficits (Table 3). The negative association between hypertension and peripheral neuropathy in year 1 was stronger after removing subjects who were taking NSAIDs in year 3 .

\section{Subjects with a History of Diabetes Mellitus}

In the diabetic patients, hypertension history, systolic pressure at rest, and pulse pressure were considered in the regression model. The final model, shown in Table 4, included history of hypertension (OR, 0.31; 95\% CI, 0.12 to 0.80), BMI (OR, 1.14; $95 \% \mathrm{CI}, 1.06$ to 1.23$)$, history of military service (OR, 2.77; 95\% CI, 1.09 to 7.04), and pulse pressure (OR, $1.03 / \mathrm{mm} \mathrm{Hg}$; 95\% CI, 1.00 to 1.05 ). Prior military service and higher BMI reduced the size of the association between pulse pressure and neuropathy.

The bivariate associations and regression model for medication use in the diabetic group are shown in Table 5. Use of calcium channel blockers was associated with greater prevalence of peripheral neuropathy. While use of $\beta$ blockers and NSAIDs were not, the number of diabetic subjects taking these medications was very minimal. No interactions were found.

\section{Discussion}

Hypertension and Idiopathic Peripheral Neuropathy

In primary care patients over 65 years of age with no history of 10 diseases known to cause peripheral neuropathy, a history of hypertension was associated with a reduced probability of peripheral neuropathy. We do not yet have an explanation for this, although it appears to be unrelated to severity of hypertension, current blood pressure, age, BMI, use of particular antihypertensive medications, use of NSAIDs, or number of primary care physician visits.

Since the study was cross-sectional, the association between hypertension and neuropathy could be non-causal or causal in either direction. For 
Table 4. Characteristics of Subjects with Diabetes Mellitus $(\mathrm{N}=110)$

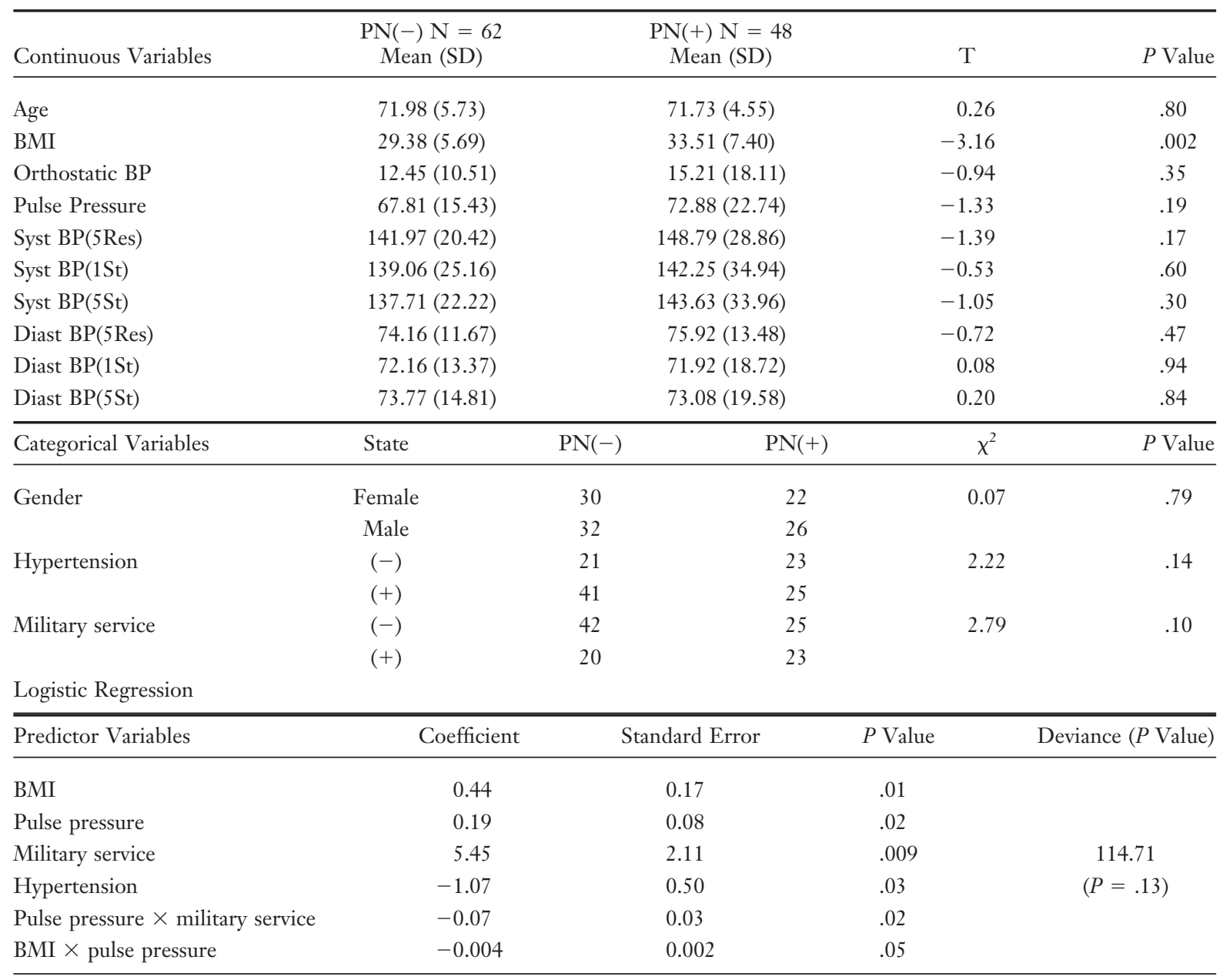

PN, peripheral neuropathy; BMI, body mass index; BP, blood pressure.

example, subjects with a history of hypertension may pay more attention to their health, see physicians more often, and lead healthier lives, and these behaviors might somehow protect them from neuropathy. There was an association between a history of hypertension and increased frequency of visits to the primary care physician. However, frequency of visits was not associated with neuropathy. Another non-causal explanation is that there was bias against selection of subjects with both hypertension and neuropathy in the cohort because of earlier disability or mortality in that group of subjects.

Causal hypotheses include a protective effect of hypertension, possibly diastolic more than systolic, ensuring more consistent perfusion of small nerve fibers. There may also be a protective effect of certain antihypertensive medications. Although no such associations were found, patients with a long history of hypertension may have been treated with a medication used more commonly in the past that protected their nerves (eg, hydrochlorthiazide or reserpine). Alternatively, perhaps peripheral neuropathy lowers blood pressure. That seems unlikely in the absence of autonomic involvement, and there was no association between orthostatic hypotension and neuropathy. Probably the most likely explanation is that hypertension is a marker for some other factor that is associated with a reduced risk for peripheral neuropathy.

We could find only 2 published studies that examined the association between hypertension and peripheral neuropathy of unknown etiology. Both found a positive rather than a negative association. The first study involved Italian primary care patients 55 years of age and older. Subjects with 2 or more symptoms of neuropathy underwent neurological examinations. Those 
Table 5. Characteristics of Subjects with Diabetes Mellitus Who Were Still Enrolled in the Third Year of the Study $(\mathrm{N}=45)$

\begin{tabular}{|c|c|c|c|c|c|}
\hline Continuous Variables & $\begin{array}{c}\mathrm{PN}(-) \mathrm{N}=25 \\
\text { Mean (SD) }\end{array}$ & \multicolumn{2}{|c|}{$\begin{array}{c}\mathrm{PN}(+) \mathrm{N}=20 \\
\text { Mean }(\mathrm{SD})\end{array}$} & $\mathrm{T}$ & $P$ Value \\
\hline Age & $71.32(5.55)$ & \multicolumn{2}{|c|}{$71.15(4.30)$} & 0.12 & .91 \\
\hline BMI & $28.97(4.77)$ & \multicolumn{2}{|c|}{$35.58(7.40)$} & -3.36 & .002 \\
\hline Pulse pressure & $63.28(16.15)$ & \multicolumn{2}{|c|}{$75.00(25.39)$} & -1.79 & .08 \\
\hline Otho BP & $14.00(10.23)$ & \multicolumn{2}{|c|}{$15.10(25.66)$} & -0.18 & .86 \\
\hline Diast BP(5 Rest) & $73.84(10.98)$ & \multicolumn{2}{|c|}{$77.20(14.38)$} & -0.86 & .39 \\
\hline Diast BP(1 Stand) & $71.76(12.80)$ & \multicolumn{2}{|c|}{$71.70(24.45)$} & 0.01 & .99 \\
\hline Diast BP(5 Stand) & $73.12(12.78)$ & \multicolumn{2}{|c|}{$71.30(24.74)$} & 0.30 & .77 \\
\hline Syst BP(5 Rest) & $137.12(19.12)$ & \multicolumn{2}{|c|}{$152.20(33.14)$} & -1.81 & .08 \\
\hline Syst BP(1 Stand) & $135.92(24.49)$ & \multicolumn{2}{|c|}{$143.10(45.15)$} & -0.64 & .53 \\
\hline Syst BP(5 Stand) & $130.16(17.59)$ & \multicolumn{2}{|c|}{$142.60(45.63)$} & -1.15 & .26 \\
\hline Categorical Variables & State & $\mathrm{PN}(-)$ & $\mathrm{PN}(+)$ & $x^{2}$ & $P$ Value \\
\hline \multirow[t]{2}{*}{ Gender } & Female & 10 & 7 & 0.12 & .73 \\
\hline & Male & 15 & 13 & & \\
\hline \multirow[t]{2}{*}{ Hypertension } & $(-)$ & 9 & 10 & 0.89 & .34 \\
\hline & $(+)$ & 16 & 10 & & \\
\hline \multirow[t]{2}{*}{ ACE-I \& ARB } & $(-)$ & 16 & 11 & 0.38 & .54 \\
\hline & $(+)$ & 9 & 9 & & \\
\hline \multirow[t]{2}{*}{$\alpha$ blocking agents } & $(-)$ & 21 & 19 & 1.36 & .24 \\
\hline & $(+)$ & 4 & 1 & & \\
\hline \multirow[t]{2}{*}{$\beta$ blocking agents } & $(-)$ & 19 & 15 & 0.01 & .94 \\
\hline & $(+)$ & 6 & 5 & & \\
\hline \multirow[t]{2}{*}{ CCB } & $(-)$ & 22 & 11 & 6.19 & .01 \\
\hline & $(+)$ & 3 & 9 & & \\
\hline \multirow[t]{2}{*}{ Diuretics } & $(-)$ & 21 & 12 & 3.27 & .07 \\
\hline & $(+)$ & 4 & 8 & & \\
\hline \multirow[t]{2}{*}{ Vasodilators } & $(-)$ & 24 & 18 & 0.64 & .42 \\
\hline & $(+)$ & 1 & 2 & & \\
\hline \multirow[t]{2}{*}{ NSAIDs } & $(-)$ & 22 & 18 & 0.05 & .83 \\
\hline & $(+)$ & 3 & 2 & & \\
\hline \multirow[t]{2}{*}{ Military service } & $(-)$ & 16 & 8 & 2.57 & .11 \\
\hline & $(+)$ & 9 & 12 & & \\
\hline \multirow[t]{3}{*}{ Number of medications } & 0 & 10 & 4 & 4.72 & .09 \\
\hline & 1 & 8 & 4 & & \\
\hline & 2 or more & 7 & 12 & & \\
\hline \multicolumn{6}{|l|}{ Logistic Regression } \\
\hline Predictor Variables & Coefficient & Standard Error & $P$ Value & & Deviance $(P$ Value $)$ \\
\hline BMI & 0.32 & 0.12 & .005 & & \\
\hline Military Service & 3.44 & 1.46 & .02 & & 27.78 \\
\hline Syst BP(5 Rest) & 0.05 & 0.02 & .02 & & $(P=.86)$ \\
\hline $\mathrm{CCB}$ & 3.28 & 1.54 & .03 & & \\
\hline
\end{tabular}

PN, peripheral neuropathy; ACE-I \& ARB, angiotensin-converting enzyme inhibitor and angiotensin receptor blocker; BMI, body mass index; NSAIDs, nonsteroidal antiinflammatory drugs. CCB, calcium channel blocker; BP, blood pressure.

with 2 bilateral neurological deficits were included in the study population. The prevalence of peripheral neuropathy in their sample was therefore 10 times lower than in ours. ${ }^{6}$ They did not control for BMI or use of $\beta$-blockers and NSAIDs, all of which were associated with both hypertension and peripheral neuropathy in our population. 
The other study examined a group of subjects in The Netherlands who had been referred to neurologists. ${ }^{5}$ It compared subjects with idiopathic peripheral neuropathy to age- and gender-matched controls and to a group of subjects with peripheral arterial disease. Hypertension was found to be associated with peripheral neuropathy in this group (OR, 2.0; 95\% CI, 1.1 to 3.6). They did not control for $\beta$-blockers or NSAIDs. BMI was not found to be associated with neuropathy in this study.

\section{Hypertension and Peripheral Neuropathy in Diabetic Subjects}

The negative association between historical hypertension and neuropathy was also found in diabetic subjects. However, pulse pressure was positively associated with peripheral neuropathy in these subjects suggesting that vascular factors may be involved, and that diabetic neuropathy and age-associated neuropathy may have different causes. The association of elevated blood pressure with peripheral neuropathy in diabetics is consistent with previous studies. ${ }^{4,5}$ The cause of diabetic peripheral neuropathy has been the subject of considerable debate between those who believe that metabolic abnormalities (eg, the polyol pathway) are responsible, and those who favor a microvascular disease mechanism. In fact, both may be involved and may be interrelated. ${ }^{8-10}$

\section{Limitations of the Study}

The study population was drawn from a primary care practice-based research network (PBRN) in Oklahoma. Although there are some differences between physicians who join PBRNs and those who do not, ${ }^{11-13}$ there is little evidence that their patients are substantially different. The 854 subjects enrolled in the larger cohort were members of a much larger group of 1836 qualified subjects. Participants were more likely than nonparticipants to be younger, male, better educated, and in better health. ${ }^{1}$ Subjects who were in a nursing home or were too confused to give informed consent were excluded. It is not clear how these selection biases and exclusion criteria may have altered the associations between hypertension-related factors and peripheral neuropathy.

Only 287 of 584 subjects remained in the study in year 3 when medications were documented. Subjects with peripheral neuropathy but not hypertension dropped out of the study at a higher rate than subjects with peripheral neuropathy and hypertension $(P=.008)$. We are not sure why this happened since the reasons for dropping out were similar. This reduces our confidence in the associations found between peripheral neuropathy and medications.

The diagnoses of hypertension, diabetes, and the 10 diseases associated with peripheral neuropathy were based on self-report. The accuracy of selfreport varies for different health conditions and in different populations. In populations with universal access to primary medical care (eg, military personnel) self-reported history of hypertension was accurate in $94 \%$ of cases when compared with medical records. ${ }^{14}$ In a study of subjects referred for cataract surgery, the accuracy of self-reported hypertension and diabetes were both above $90 \% .{ }^{15}$ However, both hypertension and diabetes are underdiagnosed in older patients. ${ }^{16}$ As a result, we may have misclassified a number of subjects with systolic hypertension and mild diabetes. In fact, of the 303 subjects with no history of hypertension, 116 had a measured systolic pressure of $>140$ but only 4 had a diastolic blood pressure of $>90$ at baseline. This could be expected to reduce the size but not the direction of the associations found.

The diagnosis of peripheral neuropathy was made entirely by physical examination. The American Academies of Neurology and Physical Medicine and Rehabilitation and the American Association of Electrodiagnostic Medicine have recently suggested a tiered definition of peripheral neuropathy. ${ }^{17}$ They concluded that physical findings were more accurate than symptoms for making the diagnosis and that multiple physical abnormalities were more reliable than individual ones. Electrodiagnostic studies are obviously more accurate, but they are impractical for epidemiologic research. Because of the care taken in training the research nurses, the strict criteria used to rate the presence or absence of abnormalities, and the nearly perfect agreement between the two nurses, we are reasonably confident that misclassification in regard to clinically apparent neuropathy status was infrequent.

We were primarily interested in patients with no obvious medical reason for neuropathy other than age. We therefore excluded subjects with a history of the more significant medical risk factors. However, just as with hypertension, some of these conditions are often underdiagnosed (eg, B12 defi- 
ciency). We did not exclude subjects with a history of some other conditions known to sometimes cause peripheral neuropathy such as hypothyroidism, cancer, chronic lung disease with hypoxia, or atherosclerotic disease of the lower extremities, and we did not collect information on some less common risk factors like porphyria and toxic exposures. We also did not evaluate each subject to determine whether a specific cause could be identified. As a result, we probably excluded some subjects who had risk factors that were not causing their neuropathy and included some subjects with neuropathy with an identifiable cause. Among adult subjects with symptomatic peripheral neuropathy, after a complete medical evaluation, a potential etiology can be found in $68 \%$ to $87 \% .^{18,19}$ However, the prevalence of idiopathic peripheral neuropathy increases substantially with advancing age.

With regard to the analyses involving medications, our conclusions must be regarded with great caution. First, we looked for associations between medication use in year 3 and peripheral neuropathy in year 1 . Whereas medications used to treat hypertension tend to be continued for long periods of time, we have no way of knowing whether their use predated the development of neuropathy. In addition, $50 \%$ of the initial eligible participants had dropped out of the study by year 3, and some groups dropped out at greater rates than others.

The positive association between $\beta$-blockers and sensory abnormalities by examination, if real, could have less to do with neuropathy than with reduction of the intensity of the deep tendon reflexes, making them more difficult to detect. ${ }^{20-22}$ The possible association between NSAIDs and neuropathy is harder to explain. There have been no published reports of NSAID-induced peripheral neuropathy. Because NSAIDs are sometimes used to treat pain associated with peripheral neuropathy, ${ }^{23}$ it is possible that subjects with neuropathy were more likely to be taking NSAIDs for this reason, although this is thought to be unlikely.

\section{Strengths of the Study}

The study has some important strengths. The population used for the analyses was reasonably large and more representative of older primary care populations than prior studies. Questionnaire data and medications were checked by the research nurses resulting in almost no missing data. There was nearly perfect agreement between nurses on phys- ical findings. Future studies should include medical record information and more complete diagnostic testing to exclude known causes of neuropathy.

\section{References}

1. Mold JW, Vesely SK, Keyl BA, Schenk JB, Roberts $M$. The prevalence, predictors, and consequences of peripheral sensory neuropathy in older subjects. J Am Board Fam Pract 2004;17:309-18.

2. Sendtner M. Molecular biology of neurotrophic factors. Baillieres Clin Neurol 1995;4:575-91.

3. Lindsay RM. Neurotrophic growth factors and neurodegenerative diseases: therapeutic potential of the neurotrophins and ciliary neurotrophic factor. Neurobiol Aging 1994;15:249-51.

4. Tesfaye S, Chaturvedi N, Eaton SEM, et al. Vascular risk factors and diabetic neuropathy. $\mathrm{N}$ Engl J Med 2005;352:341-50.

5. Forrest KYZ, Maser RE, Pambianco G, Becker DJ, Orchard TJ. Hypertension as a risk factor for diabetic neuropathy: a prospective study. Diabetes 1997;46:665-70.

6. Zarrelli MM, Amoruso L, Beghi E, Apollo F, Viesti PD, Simone P; Italian General Practitioner Study Group. Arterial hypertension as a risk factor for chronic symmetric polyneuropathy. J Epidemiol Biostat 2001;6:409-13.

7. Teunissen LL, Franssen H, Wokke JHJ, et al. Is cardiovascular disease a risk factor in the development of axonal polyneuropathy?. J Neurol Neurosurg Psychiatry 2002;72:590-5.

8. Young MJ, Veves A, Walker MG, Boulton AJM. Correlations between nerve function and tissue oxygenation in diabetic subjects: further clues to the aetiology of diabetic neuropathy? Diabetologia 1992; 35:1146-50.

9. Hogikyan RV, Wald JJ, Feldman EL, Greene DA, Halter JB, Supiano MA. Acute effects of adrenergicmediated ischemia on nerve conduction in subjects with type 2 diabetes. Metabolism 1999;48:495-500.

10. Malik RA, Newrick PG, Sharma AK, et al. Microangiopathy in human diabetic neuropathy: relationship between capillary abnormalities and the severity of neuropathy. Diabetologia 1989;32:92-102.

11. Hammersley V, Hippisley-Cox J, Wilson A, Pringle $M$. A comparison of research general practices and their patients with other practices-a cross-sectional survey in Trent. Br J Gen Pract 2002;June:463-8.

12. Stange KC. Practice-Based Research Networkstheir current level of validity, generalizability, and potential for wider application. Arch Fam Med 1993; 2:921-3.

13. Green LA, Miller RS, Reed FM, Iverson DC, Barley GW. How representative of typical practice are practice-based research networks? A report from the Ambulatory Sentinel Practice Network Inc. (ASPN). Arch Fam Med 1993;2:939-49. 
14. Page GD, France CR. Identifying hypertension using the Ohio Blood Pressure History Survey. Mil Med 2001;166:233-6.

15. Kehoe R, Wu SY, Leske MC, Chylack LT Jr. Comparing self-reported and physician-reported medical history. Am J Epidemiol 1994;139:813-8.

16. Egan BM, Lackland DT, Cutler NE. Awareness, knowledge, and attitudes of older Americans about high blood pressure: implications for health care policy, education, and research. Arch Intern Med 2003;163:681-7.

17. England JD, Gronseth GS, Franklin G, et al. Distal symmetric polyneuropathy: a definition for clinical research-report of the American Academy of Neurology, the American Association of Electrodiagnostic Medicine, and the American Academy of Physical Medicine and Rehabilitation. Neurology 2005;64: 199-207.

18. Dyck PJ, Oviatt KF, Lambert EH, et al. Chronic polyneuropathy of undetermined cause. J Neurol Neurosurg Psychiatry 1984;47:530-5.

19. Dyck PJ, Oviatt KF, Lambert EH. Intensive evaluation of referred unclassified neuropathies yields improved diagnosis. Ann Neurol 1981;10:222-6.

20. Joseph DS. The art and science of bedside diagnosis. Baltimore (MD): Urban \& Schwarzenberg; 1990. p. 501.

21. Waal-Manning HJ. Effect of propranolol on the duration of the Achilles tendon reflex. Clin Pharmacol Ther 1969;10:199-206.

22. Marsden CD, Gimlette TMD, McAllister RG, Owen DAL, Miller TN. Effect of $\beta$-adrenergic blockade on finger tremor and Achilles reflex time in anxious and thyrotoxic subjects. Acta Endocrinol 1968;57:353-62.

23. Cohen KL, Harris S. Efficacy and safety of nonsteroidal anti-inflammatory drugs in the therapy of diabetic neuropathy. Arch Intern Med 1987;147: 1442-4. 